University of Montana

ScholarWorks at University of Montana

$10-2008$

\title{
Molecular-Orientation-Dependent Ac Stark Effect and its Impact on Multiphoton Processes
}

Xi Chu

University of Montana - Missoula, xi.chu@mso.umt.edu

Follow this and additional works at: https://scholarworks.umt.edu/chem_pubs

Part of the Biochemistry Commons, and the Chemistry Commons Let us know how access to this document benefits you.

\section{Recommended Citation}

Chu, Xi, "Molecular-Orientation-Dependent Ac Stark Effect and its Impact on Multiphoton Processes" (2008). Chemistry and Biochemistry Faculty Publications. 31.

https://scholarworks.umt.edu/chem_pubs/31

This Article is brought to you for free and open access by the Chemistry and Biochemistry at ScholarWorks at University of Montana. It has been accepted for inclusion in Chemistry and Biochemistry Faculty Publications by an authorized administrator of ScholarWorks at University of Montana. For more information, please contact scholarworks@mso.umt.edu. 


\title{
Molecular-orientation-dependent ac Stark effect and its impact on multiphoton processes
}

\author{
$\mathrm{Xi} \mathrm{Chu}$ \\ Department of Chemistry, The University of Montana, Missoula, Montana 59812, USA
}

(Received 21 July 2008; published 13 October 2008)

\begin{abstract}
We study the dependence of the ac Stark shifts of electronic energies on the molecular orientation relative to the polarization direction of an incident intense laser field, using a three-dimensional non-Hermitian Floquet method and $\mathrm{H}_{2}{ }^{+}$as a model system. Simultaneously, we also study the orientation-dependent high-order harmonic generation (HHG) and multiphoton ionization (MPI). We find that with the presence of near-onephoton resonance, the Stark effect strongly mixes electronic states of different symmetries to create quasienergy states (QESs). The orientation dependence of multiphoton processes, in which these QESs play an important role, becomes complex. Population transfer is better achieved with aligned molecules and optimized orientation. When the energies of emitted photons are lower than the ionization energy, the Stark effect plays an important role in the orientation dependence of HHG intensity and polarization. It also is important for MPI, when the Keldysh parameter is larger than 1. Results from fixed-nuclei calculations for a series of internuclear distances are used to obtain values for different vibrational states. With a multiphoton resonance, the HHG intensity of the fixed-nuclei model resembles that of the lowest vibrational state. Molecular vibration of $\mathrm{H}_{2}^{+}$ has a great effect in MPI and the off-resonant HHG.

DOI: $10.1103 /$ PhysRevA.78.043408

PACS number(s): $33.80 . \mathrm{Rv}, 42.50 . \mathrm{Hz}, 33.80 . \mathrm{Eh}, 33.90 .+\mathrm{h}$
\end{abstract}

\section{INTRODUCTION}

Molecular multiphoton processes depend on the orientation of molecules relative to the polarization of the laser field [1-9]. This makes molecules different from rare-gas atoms in terms of strong field physics. There has been significant experimental and theoretical effort in studying molecular alignment and orientation-dependent multiphoton ionization (MPI) [5-7] and high-order harmonic generation (HHG) $[1-4,8,9]$. Their applications include probing the groundstate electronic structure of molecules [10-12], their proton dynamics [13], and their rotational wave packets [1]. The ionization potential and the symmetry of the highest occupied molecular orbital (HOMO) are key parameters for predicting molecule-intense-laser-field interactions [14-19]. An intense laser field, however, can alter the electronic structure in such a way that both the ionization potential and the symmetry of the HOMO depend on the orientation. Furthermore, it alters the energies and symmetries of excited states. These are all due to the ac Stark effect. The purpose of this paper is to demonstrate how the ac Stark effect affects the orientation dependence of multiphoton processes.

There are several multiphoton processes, in which both ground- and excited-state energies and symmetries are important. For instance, there is an ongoing effort to identify and to control population transfer through multiphoton excitation (ME) [20-25], which can be complicated by the orientation-dependent Stark effects. Moreover, theoretical studies on $\mathrm{C}_{60}$ [26] and aromatic molecules [27] show that electronic bound-bound transitions dominate HHG spectra. HHG peaks of $\mathrm{C}_{60}$ provide accurate transition energies, therefore $\mathrm{HHG}$ is a promising mechanism for probing electronic structures of certain molecules [26]. For $\mathrm{H}_{2}{ }^{+}$, similar harmonic peaks caused by excitation appear [28]. To obtain accurate transition energies from these peaks, however, one must resolve the orientation dependence of ac Stark shifts. When the energy of a harmonic is less than the ionization energy, the electronic structure of both the ground state and excited states and their symmetries play an important role, and is the focus of this paper. Most studies on the orientation dependence of strong field ionization focus on the situation in which the ionization is tunneling-dominant [29]. Here we study multiphoton ionization when the Keldysh parameter [30] is larger than 1, i.e., the process is not dominated by tunneling and the complex ac Stark effect on the excited states plays a role.

In this paper, we first qualitatively analyze the orientation dependence of the ac Stark effect in general terms using perturbation theory and Floquet theory. We focus on the energy shifts and the mixings of states of different symmetries. In order to study nonperturbatively the ac Stark shifts and the relevant multiphoton processes, we have developed a complex scaling non-Hermitian Floquet method. The method will be described in detail. For the numerical study, we use $\mathrm{H}_{2}{ }^{+}$as a model system because of its simplicity. The study on $\mathrm{H}_{2}{ }^{+}$ can provide reliable numerical results of a single electron system, from which models for complex systems can be built and multielectron effects can be studied in comparison. Recent calculations on this system $[28,31]$ provide us with data to calibrate our numerical method. Our numerical results and analysis identify the key factors that determine the complexity of the ac Stark effect. They also offer proof of its impact on the orientation dependence of multiphoton processes.

We also study the influence of molecular vibration on HHG and MPI. Electrons rescattered during the HHG process can be used to monitor molecular vibration due to its small de Broglie wavelength [32]. In our case, the electron rescattering model cannot be applied due to the low harmonic energy. There are previous theoretical studies on the HHG of $\mathrm{H}_{2}$ that have included the nuclear degrees of freedom but only two electronic states [33]. By including a large number of electronic states, we offer a more complete picture of both HHG and MPI and their orientation and vibration dependence. 


\section{THEORY AND COMPUTATIONAL METHODS}

The orientation dependence of ac Stark shifts can be understood qualitatively from the leading term in a timedependent perturbation theory treatment in which the field is treated as the perturbation. When there is no resonance, the shift of the electronic energy of a closed-shell atom is isotropic and, in atomic units, is given by

$$
\delta \epsilon^{(2)}=-\frac{F^{2}}{4} \alpha\left(\omega_{0}\right),
$$

where $F$ is the field strength, $\omega_{0}$ is the angular frequency of the field, and $\alpha\left(\omega_{0}\right)$ is the dynamic polarizability.

For a molecule that belongs to the point groups $C_{n h}, S_{n}$, $D_{n}, D_{n d}, D_{n h}, n>2$, or an open-shell atom, the polarizability is anisotropic and we use $\alpha_{\|}$and $\alpha_{\perp}$ to stand for the polarizability parallel and perpendicular to the $C_{n}$ axis, respectively [34]. We define the angle between the polarization direction of the field and the molecular axis as the orientation angle $\gamma$, and we write the shift as

$$
\delta \epsilon^{(2)}(\gamma)=A \cos ^{2} \gamma+B
$$

where

$$
\begin{gathered}
A=-\frac{F^{2}}{4}\left[\alpha_{\|}\left(\omega_{0}\right)-\alpha_{\perp}\left(\omega_{0}\right)\right], \\
B=-\frac{F^{2}}{4} \alpha_{\perp}\left(\omega_{0}\right) .
\end{gathered}
$$

So the shift is a quadratic function of $\cos \gamma$, as well as $F$, and the magnitude of the anisotropy depends on the anisotropy of the dipole polarizability.

When the incident laser is in the VIS/IR range, near-onephoton resonances often exist among the excited states of a variety of molecules and both the ground and the excited states of dye molecules. One photon resonance can make ac Stark shifts larger in magnitude and can to a great extent mix states of different symmetries. We consider a near-onephoton resonance of states $|1\rangle$ and $|2\rangle, i=1,2$. The energy difference between the two states is $E_{2}-E_{1}=\omega_{0}+\eta$, where $\eta$ is a small number, $\left\langle 1\left|\hat{d}_{z}\right| 2\right\rangle=z_{12},\left\langle 1\left|\hat{d}_{x}\right| 2\right\rangle=\left\langle 1\left|\hat{d}_{y}\right| 2\right\rangle=0$, and $\hat{d}_{z}$ and $\hat{d}_{x}$ are, respectively, the $z$ and $x$ components of the dipole moment operator. The Hamiltonian can be modeled by a 2 $\times 2$ Floquet matrix [35],

$$
\hat{H}_{F}=\left(\begin{array}{cc}
E_{1} & \frac{F}{2} z_{12} \cos \gamma \\
\frac{F}{2} z_{12} \cos \gamma & E_{1}+\eta_{1}
\end{array}\right) .
$$

Solutions of the equation $H_{F} \psi_{i}=\epsilon_{i} \psi_{i}$ are two quasienergy states (QESs), whose wave functions can be expressed as $\psi_{i}=c_{1 i}|1\rangle+c_{2 i}|2\rangle$. Their energies, the quasienergies, are the Stark-shifted energies. When $\left|F z_{12} \cos \gamma\right| \gg\left|\eta_{1}\right|$, the quasienergies can be approximated as

$$
\epsilon_{ \pm}=E_{1}+\frac{\eta_{1}}{2} \pm \frac{F}{2} z_{12} \cos \gamma
$$

where the ac Stark shifts are linear functions of $F \cos \gamma$. For both QESs, the populations of states $|1\rangle$ and $|2\rangle$ are nearly equal, which means that in this field, it is impossible to populate state $|1\rangle$ without populating state $|2\rangle$, and vice versa. It also lowers the symmetry of the electronic wave function.

An even more complex situation exists in which there is an additional state $|3\rangle$ that couples to $|1\rangle$, and $\left\langle 1\left|\hat{d}_{x}\right| 3\right\rangle=x_{13}$, $\left\langle 1\left|\hat{d}_{y}\right| 3\right\rangle=\left\langle 1\left|\hat{d}_{z}\right| 3\right\rangle=0$, and its energy is $E_{1}+\omega_{0}+\eta_{2}$. States $|1\rangle$ and $|3\rangle$ are also near-one-photon resonant and $\eta_{2}$ is a small number. We assume that the field is in the $x z$ plane. The Floquet Hamiltonian now becomes

$$
\hat{H}_{F}=\left(\begin{array}{ccc}
E_{1} & \frac{F}{2} z_{12} \cos \gamma & \frac{F}{2} x_{13} \sin \gamma \\
\frac{F}{2} z_{12} \cos \gamma & E_{1}+\eta_{1} & 0 \\
\frac{F}{2} x_{13} \sin \gamma & 0 & E_{1}+\eta_{2}
\end{array}\right) .
$$

When $\eta_{1}=\eta_{2}=0$, the ac Stark shifts are $\mp \frac{F}{2} \sqrt{z_{12}^{2} \cos ^{2} \gamma+x_{13}^{2} \sin ^{2} \gamma}$, and 0. The QES with ac Stark shift 0 has no projection to state $|1\rangle$. For the other two QESs, $c_{1}^{2}=c_{2}^{2}+c_{3}^{2}$ and $c_{2}^{2} / c_{3}^{2}=z_{12} / x_{13} \cot \gamma$. Here, one cannot populate one of the three states $|1\rangle,|2\rangle$, or $|3\rangle$ without populating at least one other state. Although $|2\rangle$ and $|3\rangle$ are not coupled, there is a QES that consists of only $|2\rangle$ and $|3\rangle$. For each QES, the population of states $|1\rangle,|2\rangle$, and $|3\rangle$ varies with $\gamma$.

In order to provide nonperturbative numerical results for the Stark effects and multiphoton processes, we have developed a complex scaling non-Hermitian Floquet method. We modify the previous work as published in [36] to reduce computational costs and to add components that allow us to account for different orientation angles.

Consider $\mathrm{a} \mathrm{H}_{2}^{+}$molecule placed on the $z$ axis of a Cartesian coordinate system. It interacts with a monochromatic, linearly polarized, coherent laser field of frequency $\omega_{0}$ and field strength $F$. We place the field in the $x z$ plane. The timedependent Hamiltonian can be written as

$$
\hat{H}(\mathbf{r}, t)=\hat{H}_{0}(\mathbf{r})+F \cos \omega_{0} t\left[(\cos \gamma) \hat{d}_{z}+(\sin \gamma) \hat{d}_{x}\right],
$$

where $\hat{H}_{0}$ is the field-free Hamiltonian and $\mathbf{r}$ is the electron coordinate. Since the time dependence of $\hat{H}(\mathbf{r}, t)$ is periodic, the wave function $\Psi(\mathbf{r}, t)$ can be expressed according to the Floquet theorem as [37]

$$
\Psi(\mathbf{r}, t)=e^{-i \epsilon t} \Phi(\mathbf{r}, t),
$$

where $\epsilon$ is the quasienergy and $\Phi(\mathbf{r}, t)$ is time periodic and can be expanded as 


$$
\Phi(\mathbf{r}, t)=\sum_{n=-\infty}^{\infty} \Phi_{n}(\mathbf{r}) e^{-i n \omega_{0} t}
$$

The quasienergy state Fourier component $\Phi_{n}(\mathbf{r})$ is timeindependent. We further expand $\Phi_{n}(\mathbf{r})$ as

$$
\Phi_{n}(\mathbf{r})=\sum_{j=1}^{\infty} c_{j}^{n} \chi_{j}(\mathbf{r})
$$

where $\chi_{j}$ is an eigenfunction of $\hat{H}_{0}$, i.e.,

$$
\hat{H}_{0} \chi_{j}(\mathbf{r})=E_{j} \chi_{j}(\mathbf{r}),
$$

and the $E_{j}$ is the corresponding eigenvalue. We first use a complex-scaling generalized pseudospectral method [36] to solve Eq. (12). The eigenvectors of the non-Hermitian Hamiltonian matrix satisfy the biorthogonality and binormalization relations [31], $\left\langle\chi_{j^{\prime}}^{L} \mid \chi_{j}\right\rangle=0, j^{\prime} \neq j$, and $\left\langle\chi_{j}^{L} \mid \chi_{j}\right\rangle=1$, in which $\chi_{j}^{L}(\mathbf{r})=\chi_{j}(\mathbf{r})^{*}$.

Using Eqs. (8)-(12), the time-dependent Schrödinger equation,

$$
i \hbar \frac{\partial}{\partial t} \Psi(\mathbf{r}, t)=\hat{H}(\mathbf{r}, t) \Psi(\mathbf{r}, t),
$$

can be transformed into the working equations,

$$
\begin{gathered}
\left(E_{j}-n \omega\right) c_{j}^{n}+\sum_{k} h_{j k} c_{k}^{n-1}+\sum_{l} h_{j l} c_{l}^{n+1}=\epsilon c_{j}^{n} \\
(n=0, \pm 1, \pm 2, \ldots ; j=1,2, \ldots)
\end{gathered}
$$

where

$$
h_{j k}=\frac{F}{2} z_{j k} \cos \gamma+\frac{F}{2} x_{j k} \sin \gamma
$$

and

$$
\begin{aligned}
& z_{j k}=\left\langle\chi_{j}^{L}\left|\hat{d}_{z}\right| \chi_{k}\right\rangle, \\
& x_{j k}=\left\langle\chi_{j}^{L}\left|\hat{d}_{x}\right| \chi_{k}\right\rangle .
\end{aligned}
$$

We then diagonalize the Floquet matrix to get $\epsilon$ and $c_{j}^{n}$. Because of the binormalization relation, we have $\Sigma_{n} \Sigma_{j} c_{j}^{n} c_{j}^{n}$ $=1$, i.e., without complex conjugation of one of the coefficients. The quasienergy $\epsilon$ is related to the position $E_{R}$ and width $\Gamma$ of the shifted and broadened QES as $\epsilon=E_{R}-i \Gamma / 2$. We therefore simultaneously determine the ac Stark shift and the ionization rate from $\epsilon$.

The induced dipole moment can be expressed as

$$
\mathbf{D}(t)=\left\langle\psi^{L}(\mathbf{r}, t)\left|\hat{d}_{z}\right| \psi(\mathbf{r}, t)\right\rangle \mathbf{e}_{z}+\left\langle\psi^{L}(\mathbf{r}, t)\left|\hat{d}_{x}\right| \psi(\mathbf{r}, t)\right\rangle \mathbf{e}_{x},
$$

where $\mathbf{e}_{z}$ and $\mathbf{e}_{x}$ are unit vectors and $\left[\psi^{L}(\mathbf{r}, t)\right]^{*}=\psi(\mathbf{r},-t)$, therefore

$$
\mathbf{D}(t)=\sum_{m, n} e^{-i(n-m) \omega_{0} t}\left(\mathbf{e}_{z} \sum_{j, k} c_{j}^{m} c_{k}^{n} z_{j k}+\mathbf{e}_{x} \sum_{l, q} c_{l}^{m} c_{q}^{n} x_{l q}\right) .
$$

Similarly the dipole acceleration can be written as

$$
\begin{aligned}
\mathbf{A}(t)= & \left(\left\langle\psi_{i \sigma}^{L}(\mathbf{r}, t)\left|\frac{\partial V(\mathbf{r})}{\partial z}\right| \psi_{i \sigma}(\mathbf{r}, t)\right\rangle+F \cos \gamma \cos \omega_{0} t\right) \mathbf{e}_{z} \\
& +\left(\left\langle\psi_{i \sigma}^{L}(\mathbf{r}, t)\left|\frac{\partial V(\mathbf{r})}{\partial x}\right| \psi_{i \sigma}(\mathbf{r}, t)\right\rangle+F \sin \gamma \cos \omega_{0} t\right) \mathbf{e}_{x}
\end{aligned}
$$

$$
=\sum_{m, n} e^{-i(n-m) \omega_{0} t}\left(\mathbf{e}_{z} \sum_{j, k} c_{j}^{m} c_{k}^{n} a_{j k}^{z}+\mathbf{e}_{x} \sum_{l, q} c_{l}^{m} c_{q}^{n} a_{l q}^{x}\right)
$$$$
+F \cos \gamma \cos \omega_{0} t \mathbf{e}_{z}+F \sin \gamma \cos \omega_{0} t \mathbf{e}_{x},
$$

where

$$
\begin{aligned}
& a_{j k}^{z}=\left\langle\chi_{j}^{L}\left|\frac{\partial V(\mathbf{r})}{\partial z}\right| \chi_{k}\right\rangle, \\
& a_{l q}^{x}=\left\langle\chi_{l}^{L}\left|\frac{\partial V(\mathbf{r})}{\partial x}\right| \chi_{q}\right\rangle,
\end{aligned}
$$

and $V(\mathbf{r})$ is the field-free Coulomb potential. The HHG power spectrum is the Fourier transformation of the respective time-dependent dipole moment or dipole acceleration,

$$
\begin{aligned}
\left|D\left(n \omega_{0}\right)\right|^{2} & =\left|\frac{1}{T} \int_{0}^{T} \mathbf{D}(t) e^{-i n \omega_{0} t}\right|^{2}=\frac{\left|A\left(n \omega_{0}\right)\right|^{2}}{\left(n \omega_{0}\right)^{4}} \\
& =\left|\frac{1}{T} \frac{1}{n^{2} \omega_{0}^{2}} \int_{0}^{T} \mathbf{A}(t) e^{-i n \omega_{0} t}\right|^{2},
\end{aligned}
$$

where $n$ is the harmonic order. Combing Eqs. (19) and (21), respectively, with Eq. (24) we obtain

$$
\begin{aligned}
\left|D\left(n \omega_{0}\right)\right|^{2}= & \left|\sum_{m} \sum_{j, k} c_{j}^{m+n} c_{k}^{m} z_{j k}\right|^{2}+\left|\sum_{m} \sum_{j, k} c_{j}^{m+n} c_{k}^{m} x_{j k}\right|^{2}, \\
& n=1,3, \ldots, m=0, \pm 1, \ldots, \\
\left|A\left(n \omega_{0}\right)\right|^{2}= & \left|\sum_{m} \sum_{j, k} c_{j}^{m+n} c_{k}^{m} a_{j k}^{z}\right|^{2}+\left|\sum_{m} \sum_{j, k} c_{j}^{m+n} c_{k}^{m} a_{j k}^{x}\right|^{2}, \\
& n=3,5, \ldots, m=0, \pm 1, \ldots .
\end{aligned}
$$

Because of the inversion symmetry, $D\left(n \omega_{0}\right)=0$ when $n$ is an even number.

To study the effect of molecular vibration, we perform the Floquet calculations at a series of internuclear distances and obtain the average value for a vibrational state as

$$
S_{v}=\int_{0}^{\infty} S(R) \psi_{v}^{2}(R) d R,
$$

where $v$ is the vibrational quantum number, $S(R)$ is the HHG intensity or ionization rate for internuclear distance $R, S_{v}$ is the corresponding quantity for the vibrational state, and $\psi$ is the vibrational wave function. We obtain $\psi_{v}$ by solving the Schrödinger equation for the nuclei using the ground-state potential. Numerically, we use a one-dimensional Fourier pseudospectral method [38].

For each internuclear separation, we include 80 Floquet blocks and in each Floquet block approximately 3000 electronic states, whose angular momentum projection quantum 
TABLE I. HHG power spectra of $\mathrm{H}_{2}{ }^{+}$in the ground state with $R=2 a_{0}$ in the length form $\log \left|D\left(n \omega_{0}\right)\right|^{2}$ and acceleration form $\log \left|A\left(n \omega_{0}\right)\right|^{2}$, in comparison with the length form data published in [31]. They are in atomic units. The laser wavelength is $532 \mathrm{~nm}$ and the intensity is $5 \times 10^{13} \mathrm{~W} / \mathrm{cm}^{2}$.

\begin{tabular}{lccc}
\hline \hline$n$ & $\log \left|A\left(n \omega_{0}\right)\right|^{2}$ & $\log \left|D\left(n \omega_{0}\right)\right|^{2}$ & {$[31]$} \\
\hline 3 & -8.489 & -8.489 & -8.489 \\
5 & -8.391 & -8.391 & -8.392 \\
7 & -13.188 & -13.189 & -13.363 \\
9 & -14.599 & -14.593 & -14.387 \\
11 & -14.032 & -14.033 & -14.037 \\
13 & -15.150 & -15.154 & -15.167 \\
\hline \hline
\end{tabular}

numbers range from -11 to 11 , to insure convergence. The convergence was tested at $\gamma=0^{\circ}, 45^{\circ}$, and $90^{\circ}$, with respect to the number of basis functions, the energy cutoff, the maximum angular momentum projection, and the number of Floquet blocks. The quasienergies are converged to 15 digits and the harmonic intensities to more than 4 digits at these angles. The agreement between the length and acceleration forms of HHG is an indication of the accuracy. We tabulate our results and those of Ref. [31] in Table I. In [31], a time evolution matrix is first propagated using a split operator method and then diagonalized. The agreement between the two forms and two methods is excellent.

\section{RESULTS AND DISCUSSION}

Figure 1 presents the electronic energy levels of $\mathrm{H}_{2}{ }^{+}$at $R=2 a_{0}$. ac Stark shifts, given by $\delta \epsilon_{i}(\gamma) \equiv \operatorname{Re}\left[\epsilon_{i}(\gamma)\right]-E_{i}$, for the $1 \sigma_{g}$ and $1 \sigma_{u}$ states are shown in Fig. 2 as a function of the orientation angle $\gamma$. Except when otherwise indicated, calculations presented in this paper were obtained using a model laser intensity of $5 \times 10^{13} \mathrm{~W} / \mathrm{cm}^{2}$ and wavelength of $532 \mathrm{~nm}$. The shifts in Fig. 2 are both negative and the largest magnitudes occur at $\gamma=0^{\circ}$. In comparison, we also plot the shifts according to Eq. (2) with the parameters $\alpha_{\|}\left(\omega_{0}\right)$ $=5.278$ a.u. and $\alpha_{\perp}\left(\omega_{0}\right)=1.788$ a.u. for $1 \sigma_{g}$, and $\alpha_{\|}\left(\omega_{0}\right)$ $=5.693$ a.u. and $\alpha_{\perp}\left(\omega_{0}\right)=3.292$ a.u. for $1 \sigma_{u}$. These parameters are chosen such that for $\gamma=0^{\circ}$ and $90^{\circ}$, the ac Stark shifts of the Floquet calculations are reproduced. At these angles no resonances occur, as we shall see below, and hence the results are expected to be similar to the results of a second-order perturbation calculation. There is no visible difference between the two results for the $1 \sigma_{g}$ state. For the $1 \sigma_{u}$ state, the difference is visible but small in relative magnitude. So in spite of the near-five-photon resonance between the two states, higher-order terms do not play a significant role in the ac Stark shifts. The transition energy between the Stark-shifted $1 \sigma_{g}$ and $1 \sigma_{u}$ states, i.e., $\left(E_{1 \sigma_{u}}-E_{1 \sigma_{g}}\right)+\left(\delta \epsilon_{1 \sigma_{u}}\right.$ $\left.-\delta \epsilon_{1 \sigma_{g}}\right)$, is the largest at $\gamma=0^{\circ}$ and the smallest at $\gamma=90^{\circ}$, and it can also be described by a function of the form $A \cos ^{2} \gamma+B$.

The shifts of the $1 \pi_{u}$ state are presented in Fig. 3. The solid lines correspond to the QESs $\psi_{1 \pi_{u}}(\gamma)$, for which the $1 \pi_{u}$ population, $\left|\left\langle\chi_{1 \pi_{u}}^{L} \mid \psi_{1 \pi_{u}}(\gamma)\right\rangle\right|^{2}$, is the largest at a given $\gamma$.

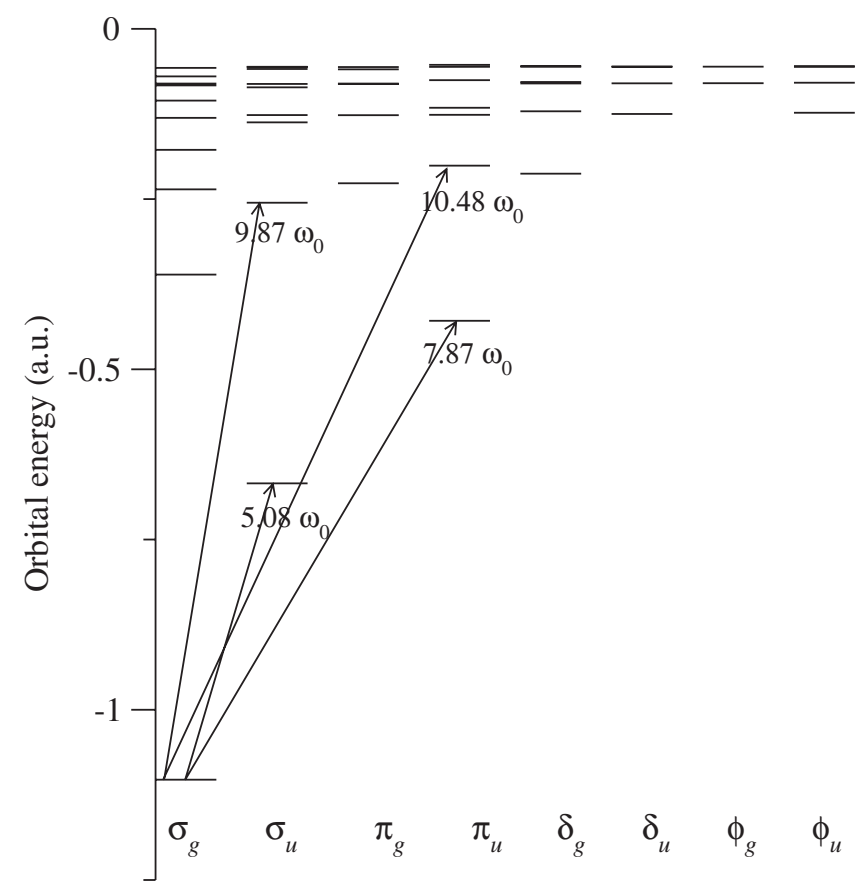

FIG. 1. The electronic energy levels of $\mathrm{H}_{2}{ }^{+}$at $R=2$ a.u. A few transition energies are labeled in terms of the photon energy of the incident laser, where $\omega_{0}=0.085645435$ a.u., which corresponds to a wavelength of $532 \mathrm{~nm}$.

There are discontinuities between $40^{\circ}$ and $41^{\circ}$, and between $51^{\circ}$ and $52^{\circ}$. We also plot in a dash-dotted line shifts of the QESs, for which the $1 \pi_{u}$ population is the second largest. A few field-free-state populations for $\psi_{1 \pi_{u}}(\gamma)$ are given in Fig. 4.

At $\gamma=0^{\circ}$, the shift is negative. The $1 \pi_{u}$ state cannot couple to $\sigma$ states, and $\left|\left\langle\chi_{1 \pi_{u}}^{L} \mid \psi(\gamma)\right\rangle\right|^{2}=0.9049$, $\left|\left\langle\chi_{1 \pi_{g}}^{L} \mid \psi(\gamma)\right\rangle\right|^{2}=0.0778$. Since there is no near-one-photon resonance with any $\pi_{g}$ states, second-order perturbation theory as in Eq. (2) can be applied when $\gamma$ is close to $0^{\circ}$. In Fig. 3, predictions of the nonperturbative Floquet method agree with Eq. (2) from $0^{\circ}$ to $30^{\circ}$.

At $\gamma=90^{\circ}$, the coupling of $1 \pi_{u}$ to $2 \sigma_{g}$ is significant, because of a near-one-photon resonance. Specifically, the popu-

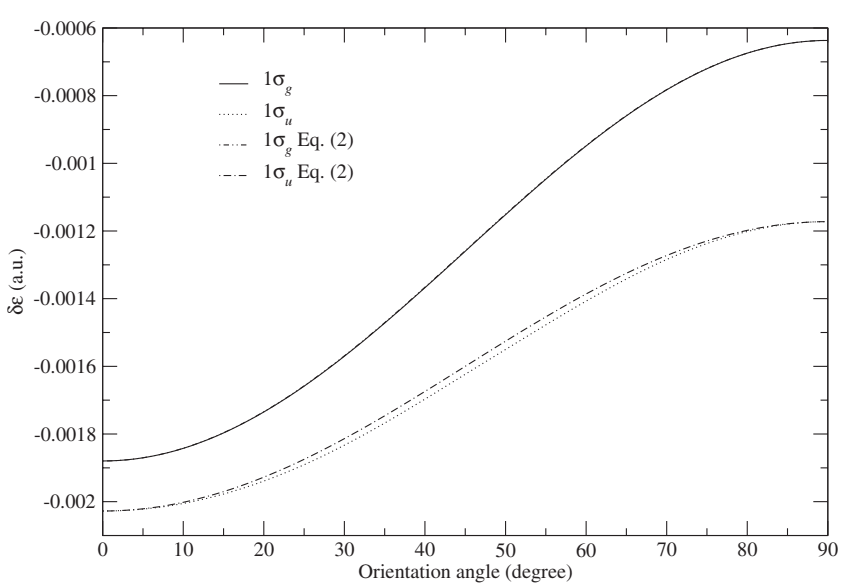

FIG. 2. The orientation-dependent ac Stark shifts of the $1 \sigma_{g}$ and $1 \sigma_{u}$ states in a $5 \times 10^{13} \mathrm{~W} / \mathrm{cm}^{2} 532 \mathrm{~nm}$ laser. 


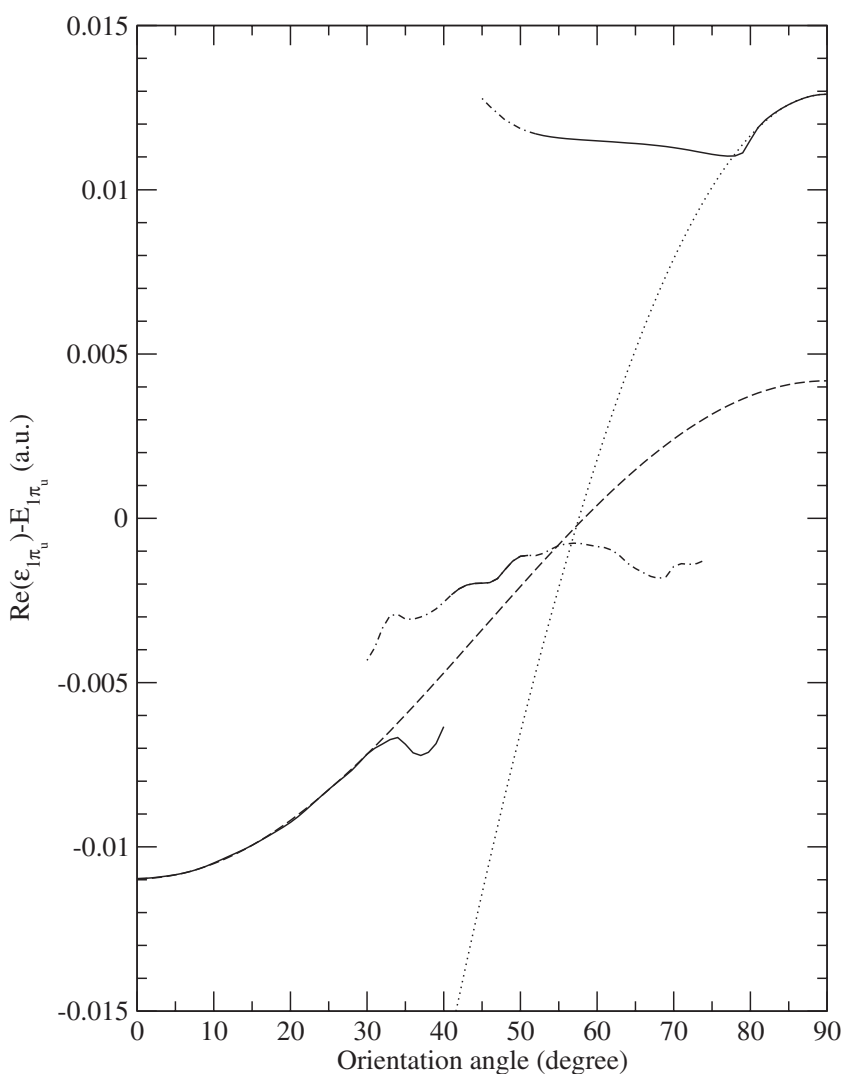

FIG. 3. The orientation-dependent ac Stark shifts of the $1 \pi_{u}$ state in a $5 \times 10^{13} \mathrm{~W} / \mathrm{cm}^{2} 532 \mathrm{~nm}$ laser. Solid line: shifts of the QES whose $1 \pi_{u}$ population is the largest. Dash-dotted line: shifts of the QES for which the $1 \pi_{u}$ population is the second largest. Dash line: predicted by $\delta \epsilon^{(2)}(\gamma)=A \cos ^{2} \gamma+B$, with $A=-0.0151529238$ and $B=0.00418555085$. Dotted line: predicted by $\delta \epsilon^{(1)}(\gamma)=A$ $+B \sin \gamma$, with $A=-0.0701276838$ and $B=0.0830375638$.

lations are $\left|\left\langle\chi_{1 \pi_{u}}^{L} \mid \psi(\gamma)\right\rangle\right|^{2}=0.3891$ and $\left|\left\langle\chi_{2 \sigma_{g}}^{L} \mid \psi(\gamma)\right\rangle\right|^{2}$ $=0.2324$. Here we can apply a formalism similar to the firstorder expression in Eq. (6), except that we use $x_{12} \sin \gamma$ to replace $z_{12} \cos \gamma$, because it is the $\hat{d}_{x}$ instead of the $\hat{d}_{z}$ operator that couples the two states. It agrees well with our nonperturbative calculations from $80^{\circ}$ to $90^{\circ}$.

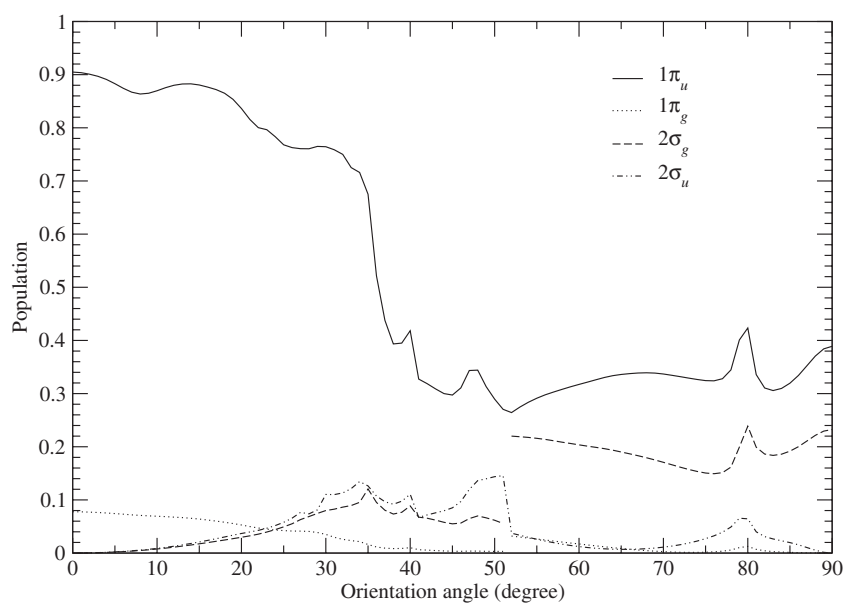

FIG. 4. A few field-free-state populations for $\psi_{1 \pi_{u}}(\gamma)$.
In between $30^{\circ}$ and $80^{\circ}$, both the parallel and the perpendicular components of the field are large enough so that $1 \pi_{u}$ couples to $2 \sigma_{g}$ and $2 \sigma_{g}$ to $2 \sigma_{u}$ with near-one-photon resonance. Therefore, the Stark effect creates a situation that resembles Eq. (7), which results in three QESs that have negative, positive, and near-zero shifts, respectively. If we keep track of the one for which the projection to $1 \pi_{u}$ is the largest, we end up having segments as the solid lines in Fig. 3.

The Stark effect mixes field-free electronic states into QES. We argue that situations in both Figs. 2 and 3 are common among the ground and excited states of molecules, and they have a profound impact on ME, HHG, and MPI.

\section{A. Multiphoton excitation}

Stark shifts are important to ME [39]. Figures 2 and 3 represent two typical cases of the excited states. Case one: as in Fig. 2, the ac Stark shifts can be described by secondorder time-dependent perturbation theory. They are relatively small in magnitude and are a function of $\cos ^{2} \gamma$. The QES mostly consists of one field-free electronic state, to which the population transfer is less likely to be complicated by the orientation dependence of the Stark effect.

Case two: as in Fig. 3, the orientation dependence of the Stark shift is complex and relatively large in magnitude. This is due to the near-one-photon resonance of at least three states of different symmetries. Due to the Stark effect, the QES has much less population in each field-free state. It is shown in Fig. 4 that in between $0^{\circ}$ and $35^{\circ}$, the $1 \pi_{u}$ population is above $70 \%$, whereas for $\gamma>35^{\circ}$, the $1 \pi_{u}$ population drops to about $30 \%$. In this case, exciting nonaligned molecules has three disadvantages: (i) The result of excitation is likely to be a mixture of field-free electronic states, which resembles the average of the QES at different $\gamma$ values. (ii) The laser can only be in multiphoton resonance for the molecules that happen to be at the right orientation. (iii) The mixing of states of different parity reduces the multiphoton transition probability from a state with well-defined parity. Using aligned molecules and optimized orientation angles can improve the excitation efficiency. In our specific example, using aligned molecules with $\gamma=33^{\circ}$ can optimize the seven-photon transition from the $1 \sigma_{g}$ to the $1 \pi_{u}$ state at $R$ $=2.0 a_{0}$.

ME from the $1 \sigma_{u}$ to the $3 \sigma_{g}$ state at $R=4.7 \AA$ has been achieved using an $800 \mathrm{~nm}$ intense laser through resonant seven-photon transition and the $3 \sigma_{g}$ population is about $1 \%$ [21]. As far as excitation efficiency is concerned, it suffers the first two disadvantages of case two. The derivation of Eqs. (5) and (6) shows that the condition for two states $|1\rangle$ and $|2\rangle$ to mix through near-one-photon resonance is $d_{12}$ $\gg d_{12}^{0}\left(F, \omega_{0}\right)$, where $d_{12}$ is the transition dipole moment and

$$
d_{12}^{0}\left(F, \omega_{0}\right)=\frac{\Delta E_{12}-\omega_{0}}{F},
$$

in which $\Delta E_{12}$ is the absolute value of the energy difference between the two states, $F$ is the field strength, and $\omega_{0}$ is the photon energy. We tabulate the comparison of $d_{12}$ and $d_{12}^{0}$ in Table II, using a field strength that corresponds to $10^{14} \mathrm{~W} / \mathrm{cm}^{2}$ intensity. These data show that a mix of five 
TABLE II. Comparison of the values of $\left(\Delta E-\omega_{0}\right) / F$ and the transition dipole moments $d$ between pairs of electronic states of $\mathrm{H}_{2}{ }^{+}$at $R=4.7 \AA, \omega_{0}=0.05696$, and $F=0.05338$. All values are in atomic units.

\begin{tabular}{lccccccc}
\hline \hline States & $3 \sigma_{g}, 1 \pi_{u}$ & $3 \sigma_{g}, 2 \sigma_{u}$ & $1 \pi_{u}, 2 \sigma_{g}$ & $1 \pi_{u}, 1 \pi_{g}$ & $2 \sigma_{u}, 1 \pi_{g}$ & $1 \sigma_{g}, 1 \sigma_{u}$ & $2 \sigma_{u}, 2 \sigma_{g}$ \\
\hline$\left(\Delta E-\omega_{0}\right) / F$ & 0.4451 & 0.9453 & 0.2990 & 0.4807 & 0.9097 & 1.033 & 0.4447 \\
$d$ & 2.121 & 6.401 & 1.789 & 3.721 & 2.148 & 4.381 & 0.5351 \\
\hline \hline
\end{tabular}

states $2 \sigma_{g}, 1 \pi_{u}, 1 \pi_{g}, 3 \sigma_{g}$, and $2 \sigma_{u}$ dominates the QESs. Aligned molecules with $\gamma=0^{\circ}$ will improve the efficiency, because at $\gamma=0^{\circ}, \sigma$ and $\pi$ states do not couple and $d_{2 \sigma_{g} 2 \sigma_{u}}$ is not significantly larger than $d_{2 \sigma_{g} 2 \sigma_{u}}^{0}$. The $3 \sigma_{g}$ and $2 \sigma_{u}$ states are therefore the only dominant components of the QESs.

\section{B. High-order harmonic generation}

The ionization energy of the ground state of $\mathrm{H}_{2}^{+}$is $12.98 \omega_{0}$ and $E_{1 \sigma_{u}}-E_{1 \sigma_{g}}=5.08 \omega_{0}$ (Fig. 1), which means that ME may play a role in the generations of the (5-11)th harmonics. Since the orientation-dependent Stark effect influences ME, it also has an impact on HHG. In Fig. 5, we plot the orientation-dependent polarization angle of these harmonics. In Fig. 6, we plot the relative intensities, $I_{n}(\gamma)$ $=\left|D\left(n \omega_{0} ; \gamma\right)\right|^{2} /\left|D\left(n \omega_{0} ; 0\right)\right|^{2}$ of each harmonic.

At $t=0$, the dipole moment vector for the $n$th harmonic can be written as

$$
\mathbf{D}\left(n \omega_{0}\right)=\left|D\left(n \omega_{0}\right)\right|\left(\cos \beta_{n} \mathbf{e}_{z}+\sin \beta_{n} \mathbf{e}_{x}\right),
$$

in which $\beta_{n}$ is what we defined as the polarization angle of the $n$th harmonic. The unit vector $\mathbf{e}_{z}$ is along the internuclear axis and the laser field vector $\mathbf{E}(t)=F \cos \omega t\left(\cos \gamma \mathbf{e}_{z}\right.$ $\left.+\sin \gamma \mathbf{e}_{x}\right)$ is in the $x z$ plane. Figure 5 tells us whether the $\sigma_{u} \rightarrow 1 \sigma_{g}$ or $\pi_{u} \rightarrow 1 \sigma_{g}$ transition dominates an emission. The polarization angle of the 5 th harmonic is very close to $0^{\circ}$, which is consistent with a $\sigma_{u} \rightarrow 1 \sigma_{g}$ transition, except for when the orientation is near $90^{\circ}$, when such a transition is no longer allowed. For the (7-11)th harmonics, the $\pi_{u} \rightarrow 1 \sigma_{g}$ transition dominates, since their polarization angles are close to $\pm 90^{\circ}$, except when $\gamma$ is around $0^{\circ}$.

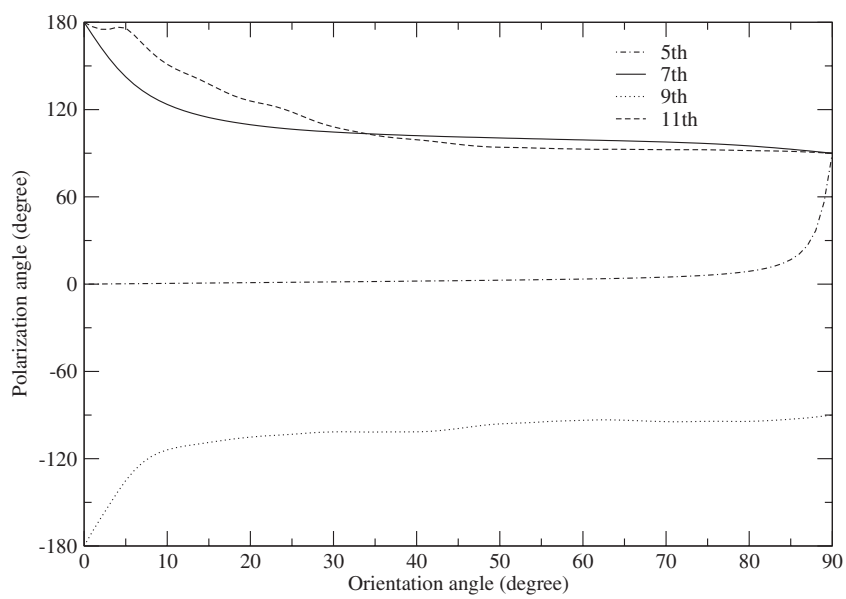

FIG. 5. The polarization of the (5-11)th harmonic of $\mathrm{H}_{2}{ }^{+}$at $R$ $=2 a_{0}$ in a $5 \times 10^{13} \mathrm{~W} / \mathrm{cm}^{2} 532 \mathrm{~nm}$ laser.
Figure 6 demonstrates that the intensity of these harmonic emissions depends strongly on the orientation angle. The 5th harmonic peaks at $0^{\circ}$ and minimizes at $90^{\circ}$. The ratio of the maximum to minimum is 6414 . The (7-11)th harmonic peak between $0^{\circ}$ and $90^{\circ}$, and the highest and lowest intensities of each harmonic, differ by more than one order of magnitude. Three factors cause the orientation dependence of these intensities: (i) symmetry of the relevant ground and excited states, (ii) the multiphoton resonance, and (iii) the ac Stark effect.

The symmetry and resonance factors dominate the 5th harmonic. Its energy and polarization indicate that its mechanism is that the system absorbs five photons to be excited to the $1 \sigma_{u}$ state, then emits one photon with an energy of $5 \hbar \omega_{0}$ to go back to the $1 \sigma_{g}$ state. Due to the symmetry, only the parallel component of the field can cause an electronic transition between the $1 \sigma_{g}$ and $1 \sigma_{u}$ states. As the orientation angle goes from $0^{\circ}$ to $90^{\circ}$, the parallel component of the


FIG. 6. The relative intensities of the (5-11)th harmonic of $\mathrm{H}_{2}^{+}$ at $R=2$ a.u. using a $5 \times 10^{13} \mathrm{~W} / \mathrm{cm}^{2} 532 \mathrm{~nm}$ laser. 
driving field decreases and so does the intensity of this emission.

The near-five-photon resonance makes the ratio of the highest to the lowest intensities large, specifically $I\left(0^{\circ}\right) / I\left(90^{\circ}\right)=6414$. In comparison, we perform similar calculations with an $800 \mathrm{~nm}$ laser, in which case $E_{1 \sigma_{u}}-E_{1 \sigma_{g}}$ $=7.63 \omega_{0}$. The 7th and 9th harmonics here have similar dependence on $\gamma$ as the 5th shown in Fig. 6. However, since they are off-resonance, $I\left(0^{\circ}\right) / I\left(90^{\circ}\right)$ is reduced to 347.5 for the 7 th, and further reduced to 60.67 for the 9 th.

The ac Stark effect, which creates both energy shifts and the mixing of states, determines the orientation dependence of the intensities of the (7-11)th harmonic. Without the Stark effect, the symmetry factor alone makes a transition between a $\pi_{u}$ and a $\sigma_{g}$ state peak at $90^{\circ}$ and minimize at $0^{\circ}$, which is not the case for these harmonics.

The energy of the 7th harmonic is close to the energy difference of the $1 \pi_{u}$ and the $1 \sigma_{g}$ states, which is $7.87 \omega_{0}$ (Fig. 1). Its intensity has a single peak at $33^{\circ}$, which indicates the relevance of the QES, i.e., the system is excited to the QES, whose wave function depends on the orientation, then emits the 7 th harmonic and returns to the ground state. The peak location is a balance of two factors: on the one hand, the $\sigma_{g}-\pi_{u}$ transition probability increases with a larger perpendicular component of the field; on the other hand, the $1 \pi_{u}$ population in the QES drops significantly for $\gamma>35^{\circ}$ (Fig. 4). The 9th and 11th harmonics have multiple peaks, which suggests that they are related to multiple QESs with multiple components that depend on $\gamma$.

Figure 7 shows the intensities of the 5th and 7 th harmonics at different internuclear distances. For the 5 th harmonic, the resonance makes the intensity larger for $R=2 a_{0}$ than when $R$ is slightly larger or smaller than 2 (except when $\gamma$ $=90^{\circ}$ and the resonance no longer exists). When $R$ is significantly larger, e.g., $R=2.85 a_{0}$ as shown in Fig. 7 , the intensity becomes much larger. For the 7 th harmonic, resonances never occur for any of the internuclear distances shown. With the exception of certain orientation angles, the intensity increases with increasing $R$. We also plot the intensities for the $v=0$ vibrational state. Our value for the zero-point energy is $1152.1 \mathrm{~cm}^{-1}$. For the 5 th harmonic, i.e., with resonance, the magnitude is fairly close to the results of fixed nuclear calculations for $R=2 a_{0}$, whereas for the 7th harmonic, i.e., without resonance, the intensity is more than two orders of magnitude larger for $v=0$ vibrational state than the $R=2 a_{0}$ values.

The power spectra of the (5-11)th harmonic for the three lowest vibrational states are presented in Fig. 8. The energy difference between $v=1$ and 0 is $2189.7 \mathrm{~cm}^{-1}$ and that between $v=2$ and 1 is $2063.7 \mathrm{~cm}^{-1}$. Larger energy gap roughly correlates with larger intensity gap. This is because with larger vibrational energy, there is a higher probability of larger internuclear distances, which produce higher HHG intensities in most cases (Fig. 7).

\section{Multiphoton ionization}

There is little ionization of the $1 \sigma_{g}$ state with $R=2 a_{0}$ using our laser parameters. Its ionization rate for the first low-
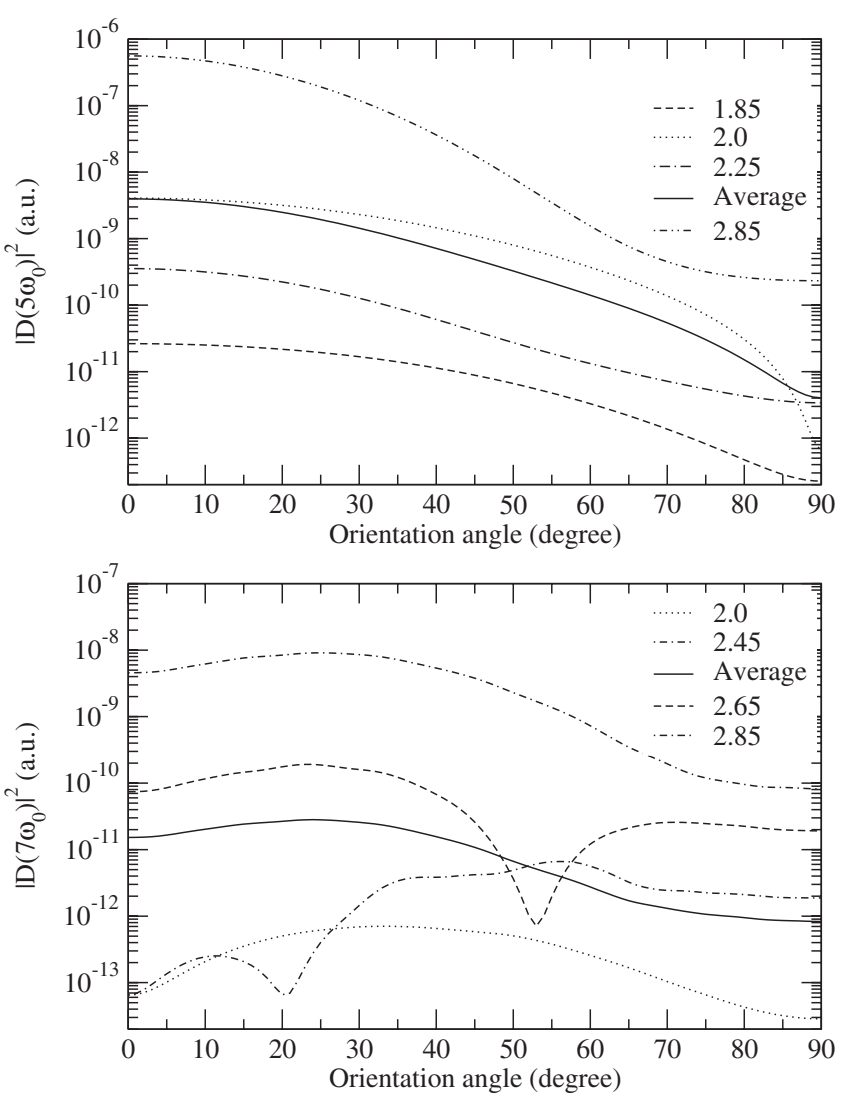

FIG. 7. The intensities of the 5th and 7th harmonic of $\mathrm{H}_{2}^{+}$at various internuclear distances using a $5 \times 10^{13} \mathrm{~W} / \mathrm{cm}^{2} 532 \mathrm{~nm}$ laser and the average values corresponding to a $v=0$ vibrational state.

est vibrational states are presented in Fig. 9. The three curves have similar shapes, and larger vibrational energy corresponds to larger ionization probabilities. The anisotropies are large. This is because a multiphoton dominant ionization process depends strongly on the electronic structure, particularly the ground and low-lying excited states. Here, the Keldysh parameter for $R=2 a_{0}$ is 3.4 and the electronic structure of $\mathrm{H}_{2}{ }^{+}$(Fig. 1) makes it extremely hard to ionize when the $\gamma$ $=90^{\circ}$. The ratio of $\Gamma\left(\gamma=0^{\circ}\right) / \Gamma\left(\gamma=90^{\circ}\right)$ for $v=2$, however, is smaller than for $v=0$, because the ionization rates for larger internuclear distances weigh more for $v=2$ and they are much larger in magnitude and smaller in anisotropy. When the process becomes tunneling dominant, i.e., the intensity is larger than $6 \times 10^{14} \mathrm{~W} / \mathrm{cm}^{2}$, we expect the anisotropy to be even smaller.

The ionization rates of the $1 \sigma_{u}$ and $1 \pi_{u}$ states are plotted in Figs. 10 and 11. The Keldysh parameter [30], defined as $\kappa=\sqrt{I_{p} / 2 U_{p}}$ with $U_{p}=F^{2} / 4 \omega_{0}$, is larger than 1 for these two states. This indicates that the ionization is a multiphotondominant, rather than a tunneling dominant process. The purpose of presenting the ionization data of these states is not to study the MPI of the excited states, but only to use the $1 \sigma_{u}$ and the $1 \pi_{u}$ states to model what happens to MPI with and without the presence of near-one-photon resonant coupling.

The ionization rate of the $1 \sigma_{u}$ state shown in Fig. 10 has a shape similar to Telnov and Chu's [28] results using an $800 \mathrm{~nm}$ laser of the same intensity. With a larger intensity, i.e., smaller $\kappa$ value, the ionization peak occurs at $\gamma=0^{\circ}$ 


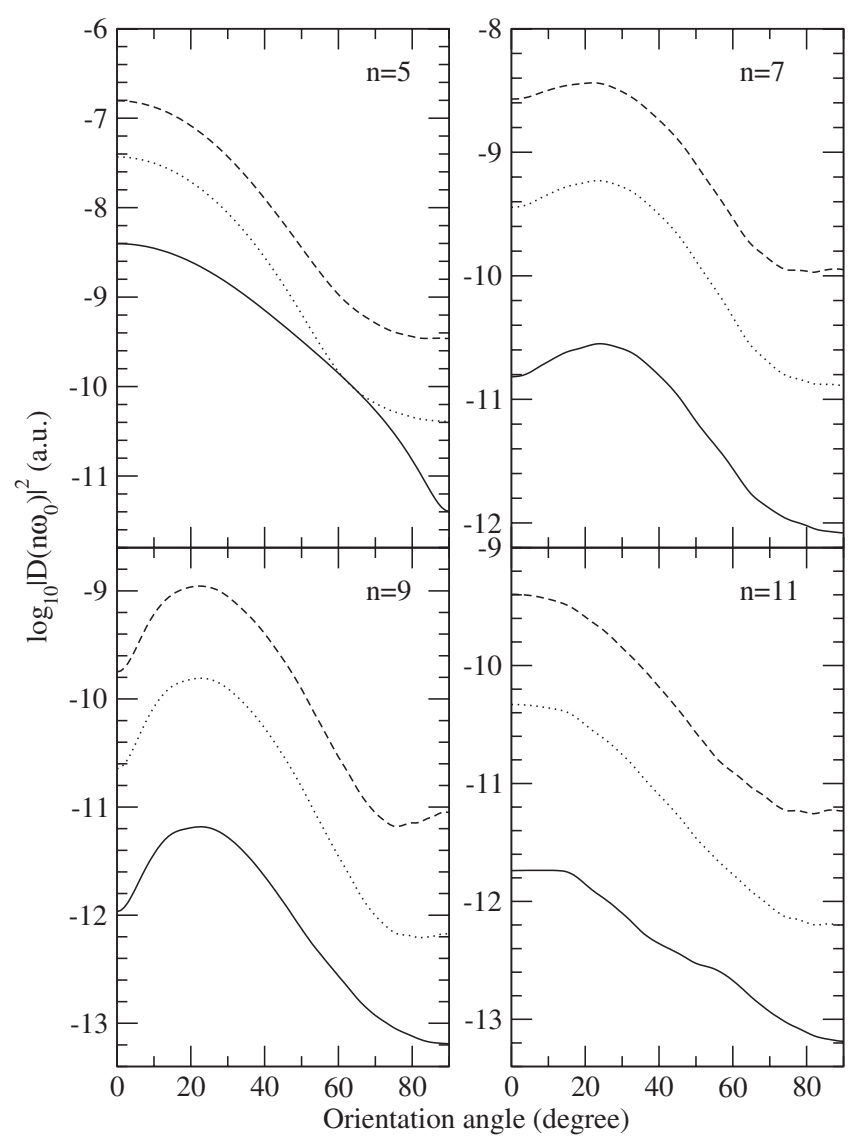

FIG. 8. Power spectra of the (5-11)th harmonic of $\mathrm{H}_{2}^{+}$using a $5 \times 10^{13} \mathrm{~W} / \mathrm{cm}^{2} 532 \mathrm{~nm}$ laser. Solid lines: $v=0$, dotted lines: $v=1$, and dash lines: $v=2$ vibrational states.

[28,40]. In Fig. 10, the peak occurs at $\gamma=36^{\circ}$, because in a multiphoton-dominant process, excited states play a more significant role. The Stark effect mixes excited states of $\sigma, \pi$, and $\delta$ symmetry and makes it easier for such a QES to couple to higher excited states and the continuum. Due to the mixing of symmetries, both the parallel and perpendicular components of the field are needed to enhance ionization. The ionization rate of the $1 \pi_{u}$ state shown in Fig. 11 has

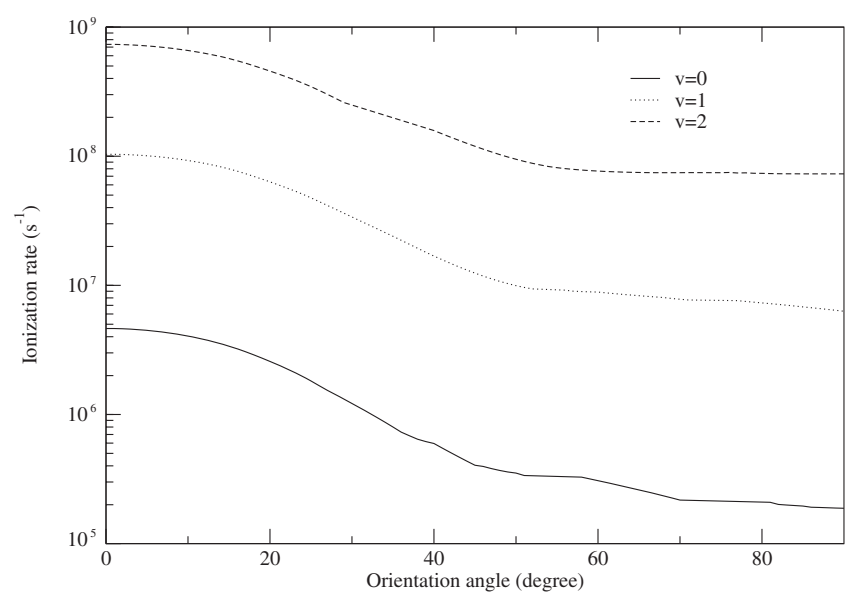

FIG. 9. The ionization rate of $\mathrm{H}_{2}{ }^{+}$for different vibrational states using a $5 \times 10^{13} \mathrm{~W} / \mathrm{cm}^{2} 532 \mathrm{~nm}$ laser.

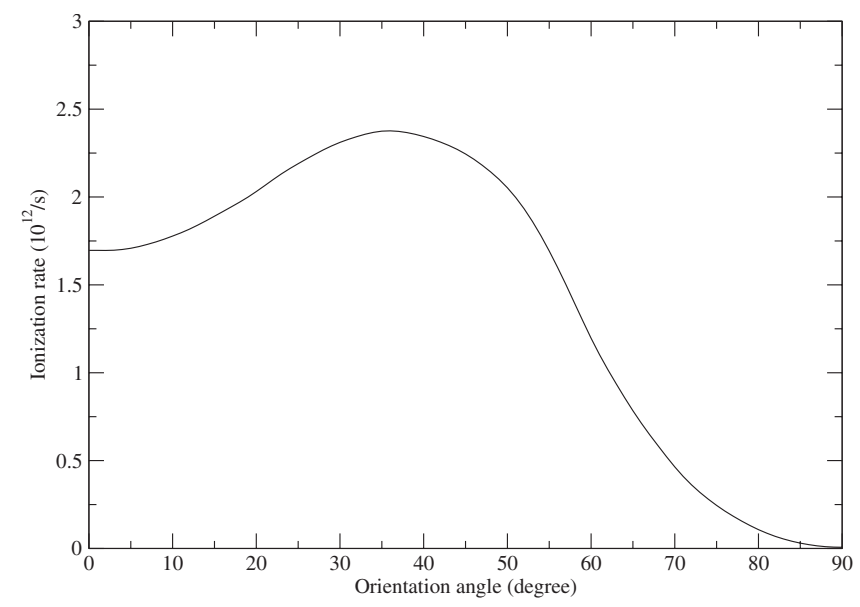

FIG. 10. The ionization rate of the $1 \sigma_{u}$ state of $\mathrm{H}_{2}{ }^{+}$at $R=2 a_{0}$ in a $5 \times 10^{13} \mathrm{~W} / \mathrm{cm}^{2} 532 \mathrm{~nm}$ laser.

discontinuities, because at different angles three different QESs have the largest $1 \pi_{u}$ population. Figure 11 describes how the three QESs behave in terms of ionization. It demonstrates that MPI can have a complex orientation dependence if the state is in near-one-photon resonance with other states.

\section{CONCLUSION}

Using the three dimensional nonperturbative Floquet method that we developed, we have studied the orientationdependent ac Stark shifts of the ground and excited states of $\mathrm{H}_{2}^{+}$and their ionization rate. We have also studied the orientation-dependent polarizations and intensities of HHG.

Without one-photon resonance, the ac stark shifts are relatively small in magnitude and can be described by $a^{2} \cos ^{2} \gamma$ function. With near-one-photon resonances, the Stark effect mixes states of different symmetry and the shifts can have a complex orientation dependence. To achieve population transfer to such an excited state, using aligned molecules and optimized orientation angles may improve the population transfer efficiency.

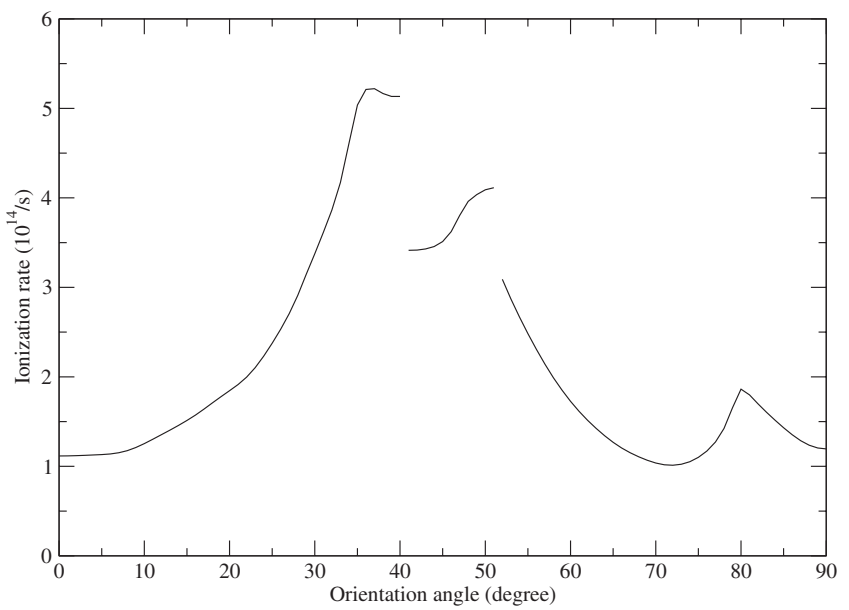

FIG. 11. The ionization rate of the $1 \pi_{u}$ state of $\mathrm{H}_{2}{ }^{+}$at $R=2 a_{0}$ in a $5 \times 10^{13} \mathrm{~W} / \mathrm{cm}^{2} 532 \mathrm{~nm}$ laser. 
When the energy of a harmonic emission is less than the ionization energy, three factors determine the orientation dependence of the HHG intensity: the symmetry of the relevant electronic states, multiphoton resonance, and the ac Stark effect. The symmetry and the Stark effect determine at which orientation angle the intensity peaks or minimizes. They also determine the orientation-dependent polarization of a given harmonic. The multiphoton resonance enhances the ratio of the highest intensity to lowest intensity of the orientationdependent harmonic emission.

Both the ground and the excited states and their ac Stark shifts are important to MPI when the process is multiphotondominant. A multiphoton dominant process, therefore, can have a more complex orientation dependence than a tunneling-dominant process.

Molecular vibration has a large effect on the multiphoton processes we studied in this paper. The small nuclear mass of
$\mathrm{H}_{2}^{+}$makes the probability of larger nuclear separation higher. Without multiphoton resonance, larger internuclear distance gives higher HHG intensity and larger ionization rate. With resonance, however, the HHG intensity of the $v$ $=0$ vibrational state can be close to the value of a fixed nuclei calculation with the electronic structure at which the resonance happens.

\section{ACKNOWLEDGMENTS}

I acknowledge the discussions and suggestions of Dr. Gerrit Groenenboom and Dr. Phil Stimac. This work is supported by National Science Foundation Grant No. EPS0346458 and the Office of the Vice President for Research and Development of the University of Montana.
[1] S. Ramakrishna and T. Seideman, Phys. Rev. Lett. 99, 113901 (2007).

[2] A. T. Le, X. M. Tong, and C. D. Lin, J. Mod. Opt. 54, 967 (2007).

[3] C. B. Madsen, A. S. Mouritzen, T. K. Kjeldsen, and L. B. Madsen, Phys. Rev. A 76, 035401 (2007).

[4] X. X. Zhou, X. M. Tong, Z. X. Zhao, and C. D. Lin, Phys. Rev. A 72, 033412 (2005).

[5] I. V. Litvinyuk, K. F. Lee, P. W. Dooley, D. M. Rayner, D. M. Villeneuve, and P. B. Corkum, Phys. Rev. Lett. 90, 233003 (2003).

[6] T. K. Kjeldsen, C. Z. Bisgaard, L. B. Madsen, and H. Stapelfeldt, Phys. Rev. A 68, 063407 (2003).

[7] V. Kumarappan, L. Holmegaard, C. Martiny, C. B. Madsen, T. K. Kjeldsen, S. S. Viftrup, L. B. Madsen, and H. Stapelfeldt, Phys. Rev. Lett. 100, 093006 (2008).

[8] T. Kanai, S. Minemoto, and H. Sakai, Nature 435, 470 (2005).

[9] J. Itatani, D. Zeidler, J. Levesque, M. Spanner, D. M. Villeneuve, and P. B. Corkum, Phys. Rev. Lett. 94, 123902 (2005).

[10] J. Itatani, J. Levesque, D. Zeidler, H. Niikura, H. Pepin, J. Kieffer, P. Corkum, and D. Villeneuve, Nature 432, 867 (2004).

[11] S. Patchkovskii, Z. Zhao, T. Brabec, and D. M. Villeneuve, Phys. Rev. Lett. 97, 123003 (2006).

[12] R. Torres et al., Phys. Rev. Lett. 98, 203007 (2007).

[13] S. Baker, J. S. Robinson, C. A. Haworth, H. Teng, R. A. Smith, C. C. Chiril, M. Lein, J. W. G. Tisch, and J. P. Marangos, Science 312, 424 (2006).

[14] X. M. Tong, Z. X. Zhao, and C. D. Lin, Phys. Rev. A 66, 033402 (2002).

[15] J. Muth-Bohm, A. Becker, and F. H. M. Faisal, Phys. Rev. Lett. 85, 2280 (2000).

[16] J. Muth-Bohm, A. Becker, S. L. Chin, and F. H. M. Faisal, Chem. Phys. Lett. 337, 313 (2001).

[17] T. Brabec, M. Cote, P. Boulanger, and L. Ramunno, Phys. Rev. Lett. 95, 073001 (2005).

[18] K. Mishima, K. Nagaya, M. Hayashi, and S. H. Lin, Phys. Rev.
A 70, 063414 (2004).

[19] K. Nagaya, K. Mishima, M. Hayashi, and S. Lin, Chem. Phys. Lett. 424, 34 (2006).

[20] A. S. Alnaser, M. Zamkov, X. M. Tong, C. M. Maharjan, P. Ranitovic, C. L. Cocke, and I. V. Litvinyuk, Phys. Rev. A 72 , 041402(R) (2005).

[21] G. N. Gibson, L. Fang, and B. Moser, Phys. Rev. A 74, 041401(R) (2006).

[22] G. N. Gibson, R. N. Coffee, and L. Fang, Phys. Rev. A 73, 023418 (2006).

[23] A. Staudte et al., Phys. Rev. Lett. 98, 073003 (2007).

[24] S. Zou, Q. Ren, G. G. Balint-Kurti, and F. R. Manby, Phys. Rev. Lett. 96, 243003 (2006).

[25] C. Trallero-Herrero, D. Cardoza, T. C. Weinacht, and J. L. Cohen, Phys. Rev. A 71, 013423 (2005).

[26] G. P. Zhang, Phys. Rev. Lett. 95, 047401 (2005).

[27] R. Baer, D. Neuhauser, P. R. Zdanska, and N. Moiseyev, Phys. Rev. A 68, 043406 (2003).

[28] D. A. Telnov and Shih-I. Chu, Phys. Rev. A 76, 043412 (2007).

[29] T. K. Kjeldsen and L. B. Madsen, J. Phys. B 37, 2033 (2006).

[30] L. V. Keldysh, Sov. Phys. JETP 20, 1307 (1965).

[31] D. A. Telnov and Shih-I. Chu, Phys. Rev. A 71, 013408 (2005).

[32] N. L. Wagner, A. Wuest, I. P. Christov, T. Popmintchev, X. Zhou, M. M. Murnane, and H. C. Kapteyn, Proc. Natl. Acad. Sci. U.S.A. 103, 13279 (2006).

[33] N. Moiseyev, M. Chrysos, O. Atabek, and R. Lefebvre, J. Phys. B 28, 2007 (1995).

[34] Richard P. Leavitt, J. Chem. Phys. 72, 3472 (1979).

[35] J. H. Shirley, Phys. Rev. 138, B979 (1965).

[36] X. Chu and Shih-I. Chu, Phys. Rev. A 63, 013414 (2000).

[37] S. I. Chu, Adv. At. Mol. Phys. 21, 197 (1985).

[38] G. Yao and S. I. Chu, Chem. Phys. Lett. 204, 381 (1993).

[39] G. N. Gibson, Phys. Rev. Lett. 89, 263001 (2002).

[40] G. Lagmago Kamta and A. D. Bandrauk, Phys. Rev. A 74, 033415 (2006) 